\title{
Effect of Antibiotic Combinations on the Sensitivity of Carbapenem Resistant Acinetobacter baumannii Strains
}

\section{Elhabibi $\mathrm{T}^{1 *}$ and Ramzy $\mathrm{S}^{2}$}

${ }^{1}$ Department of Microbiology and Immunology, Faculty of Pharmacy, Egyptian Russian University, Egypt

${ }^{2}$ Department of Microbiology and Immunology, Faculty of Pharmacy, Al-Azhar University, Egypt

\begin{abstract}
Acinetobacter baumannii is one of the predominant pathogens involved in hospital acquired infections worldwide. Despite intensive surveillance and preventive measures, these infections are major cause of high mortality rates. In this study a total of $375 \mathrm{~A}$. baumannii isolates were isolated from a variety of clinical collected from 5 different hospitals in Great Cairo. These isolates were identified as A. baumannii biochemically, by API20E system and genetically by detection of $16 \mathrm{~S}$ rRNA gene. All isolates showed positive results and confirmed for the presence of the suspected gene by PCR.

The antibiotic susceptibility patterns for the isolated $A$. baumannii were also evaluated. The detection of the presence of OXA-type (OXA 23, 24, 51 and 58) carbapenemases-encoding genes which are responsible for carbapenem resistance was also performed. I observed the presence of OXA 23 gene in $84 \%$ of the isolates. While $35.2 \%$ were positive to OXA 24 gene and $87.2 \%$ were positive to OXA 51 gene. No isolates showed positive results for the presence of OXA 51 gene.

They were also evaluated for the effectiveness of drug combination of carbapenems and colistin against 30 isolates. Regarding imipenem and colistin combination, $13.3 \%$ of the strains showed synergy, while $86.7 \%$ showed additive results. For the combination of meropenem and colistin, $66.7 \%$ of the strains showed synergy, while $33.3 \%$ showed additive results. The antibacterial effect of both combinations on A. baumannii showed commonly synergistic or additive results. To a significant extent meropenem and colistin showed a superior synergy compared to imipenem and colistin.
\end{abstract}

Keywords: Acinetobacter baumannii; Carbapenem; Colistin; Carbapenemases; Synergy

\section{Introduction}

Healthcare associated infections (HAIs) are a type of infections caused by prolonged hospital stay and it accounts for a major risk factor for serious health issues leading to death [1]. About 75\% of the burden of these infections is present in developing countries [2].

Healthcare-associated infections are a worldwide dilemma, with significant mortality. For decades, Gram-negative bacilli (GNB) sustained their share of HAIs; however Acinetobacter is the main GNB associated with consistently increasing proportions of HAIs. A. baumannii is expected to become a chief cause of HAIs in Egyptian hospitals due to the extensive use of antimicrobials in intensive care units (ICUs), in addition to its inconceivable ability to acquire resistance [3].

A surveillance program conducted in Egypt showed high incidence of ICU-onset HAIs and a high resistance rates among organisms causing nosocomial infections, considered a main risk to patient safety [4]. Nonlactose fermenting bacteria such as A. baumannii species are major causes of hospital-acquired infections [5]. The prevalence of MDR A. baumannii isolates from intensive care units among hospitals in Egypt is increasing rapidly [6].

Multiple mechanisms have been implicated in the resistance of Acinetobacter spp. and it is considered one of the most virulent MDR pathogens. Acinetobacter has been isolated in food and inanimate objects and can colonize humans and live in dry or moist conditions [7].

Farid et al. [5] stated that one of the predominant Gram-negative bacteria causing HAIs in Egyptian hospitals is A. baumannii. Their infections are difficult to treat due to limited susceptibility to antimicrobial drugs and the appearance of antibiotic resistance during therapy. Multidrug resistance, which is produced by a variety of resistance mechanisms, leaves inadequate alternatives for treatment in many patients. For this reason there are not many choices available for treatment and control of such dangerous organism in Egyptian hospitals. Combination therapy is one of the supreme effective methods for treatment as it reduces adverse effects and resistance to the antibiotics used. The aim of this study is detection of A. baumannii isolates from intensive care units in Egypt. Furthermore, examine their antimicrobial susceptibility patterns and their major resistance mechanisms. Also, determine the effect of combination therapy on treating such pathogen.

\section{Materials and Methods}

\section{Bacteriological examination}

A total of 375 A. baumannii isolates were isolated from various clinical specimens including 73 blood, 67 throat swaps, 57 urine, 49 pus, 46 wound, 24 Nasogastric tube, 22 endotracheal tubes, 22 sputum and 15 nasal swaps collected from 5 health care institution in Cairo and Giza (National Cancer Institute, Al-Kasr eleiny Hospital, Al-Zahraa Hospital, Al-Demerdash Hospital, Al-Galaa Hospital). These isolates identified biochemically by using catalase test and ability to grow at $44^{\circ} \mathrm{C}[8]$ and were confirmed by API 20E test kit (BioMèrieux, France).

*Corresponding author: Elhabibi T, Department of Microbiology and Immunology, Faculty of Pharmacy, Egyptian Russian University, Egypt, Tel: 201004622554/201021411433; E-mail: tamer_elhabibi@yahoo.com

Received May 28, 2016; Accepted June 08, 2017; Published June 15, 2017

Citation: Elhabibi T, Ramzy S (2017) Effect of Antibiotic Combinations on the Sensitivity of Carbapenem Resistant Acinetobacter baumannii Strains. J Microb Biochem Technol 9:132-137. doi: 10.4172/1948-5948.1000356

Copyright: @ 2017 Elhabibi T, et al. This is an open-access article distributed under the terms of the Creative Commons Attribution License, which permits unrestricted use, distribution, and reproduction in any medium, provided the original author and source are credited. 


\section{DNA extraction}

DNA was extracted from the microbial colonies using the QIAmp DNA mini kit (Qiagen Inc.) according to the producer's recommendation. The DNA concentration and purity were determined by assessing the absorbance at $260 \mathrm{~nm}$ and by computing the ratio of absorbance at $260 \mathrm{~nm}$ to that at $280 \mathrm{~nm}$ using a spectrophotometer (U.V-VIS.), U.V 2500 (Labomed. Inc.) [9]. DNA was used directly or stored at $-20^{\circ} \mathrm{C}$ for future use.

Detection of $A$. baumannii group specific 16S rRNA gene and OXA genes (OXA 23, OXA 24, OXA 51 and OXA 58) responsible for carbapenem resistance by Multiplex PCR.

Amplification and detection of $A$. baumannii group specific gene was performed according to the technique formerly described by Misbah et al. [10]. All oligonucleotides were synthesized in Bio Basic Inc. (Canada). The sequences of the primers used for detection of $A$. baumannii group specific gene "16S rRNA" is: 5'-AGAGTTTGATCCTGGCTCAG-3' and 5'-TACCAGGGTATCTAATCCTGTT-3'. The sequences of the primers used for detection of the $O X A 23$, 24, 51 and 58 genes are: 5'-GATCGGATTGGAGAACCAGA-3' and 5'-ATTTCTGACCGCATTTCCA-3' for OXA 23, 5'-GGTTAGTTGGCCCCCTTAAA-3' and 5'-AGTTGAGCGAAAAGGGGATT-3' for OXA 24 and 5'-TAATGCTTTGATCGGCCTTG-3' and 5'-TGGATTGCACTTCATCTTGG-3' for $O X A 51$ and 5'-AAGTATTGGGGCTTGTGCTG-3' and 5'-CCCCTCTGCGCTCTACATAC-3' for OXA 58 gene primers (Kapa, USA) [11].

The PCR was made in a total volume of $25 \mu \mathrm{l}$ reaction mixtures containing $150-200 \mathrm{ng}$ of DNA as template, $0.5 \mu \mathrm{M}$ of each primer and 1x of PCR master mix (Taq Master/High yield, Jena Bioscience) which provides 2.5 units per reaction of DNA polymerase, $0.2 \mathrm{mM}$ of each deoxynucleotide triphosphate, $1 \mathrm{x}$ PCR buffer (with $1.5 \mathrm{mM}-\mathrm{MgCl}_{2}$ ). The amplification cycles were performed out in a programmable heating block, (Primus Thermal Cycler, MWG Biotech, Germany). Reaction conditions were optimized to be $94^{\circ} \mathrm{C}$ for $3 \mathrm{~min}$ as initial denaturation, followed by 30 cycles of $95^{\circ} \mathrm{C}$ for $30 \mathrm{~s}\left(55^{\circ} \mathrm{C}\right.$ for $60 \mathrm{~s}$ and $52^{\circ} \mathrm{C}$ for 40 $\mathrm{s}$ for detection of $\mathrm{A}$. baumannii and $O X A$ genes, respectively). A final extension step at $72^{\circ} \mathrm{C}$ for $5 \mathrm{~min}$ was followed. Negative control (no template) and positive control (reference strain) were included. Amplification products were electrophoresed in $2 \%$ agarose gel in $0.5 \mathrm{x}$ TBE (Tris-borate-EDTA) at $70 \mathrm{~V}$ for $60 \mathrm{~min}$ and visualized under ultraviolet light. To assure that the amplification products were of the expected size, a 1500 bp DNA ladder was run simultaneously as a marker. Presence of 750 bp, 501 bp, 249, 353 and 599 bp DNA fragment indicated positive sample of $A$. baumannii group specific OXA 23, 24, 51 and 58 genes, respectively.

\section{Antimicrobials susceptibility testing}

A total of 375 A. baumannii isolates were examined for their sensitivity to several antimicrobial agents by disc diffusion method according to Clinical laboratory standards institute [12]. Isolates were cultured on Mueller-Hinton agar and tested for their susceptibility to 12 antimicrobial agents.

The following antimicrobial discs were used: piperacillin $(100 \mu \mathrm{g})$, piperacillin/tazobactam $(75 / 10 \mu \mathrm{g})$, ampicillin- sulbactam $(10 / 10 \mu \mathrm{g})$, ceftazidime $(30 \mu \mathrm{g})$, cefepime $(30 \mu \mathrm{g})$, cefotaxime $(30 \mu \mathrm{g})$, ceftriaxone $(30 \mu \mathrm{g})$, imipenem $(10 \mu \mathrm{g})$, meropenem (10), colistin $(50 \mu \mathrm{g})$, gentamicin $(10 \mu \mathrm{g})$, tobramycin $(10 \mu \mathrm{g})$, amikacin $(30 \mu \mathrm{g})$, tetracycline $(30 \mu \mathrm{g})$, ciprofloxacin $(5 \mu \mathrm{g})$, levofloxacin $(5 \mu \mathrm{g})$ and trimethoprim/ sulfamethoxazole $(1.25 / 23.75 \mu \mathrm{g})$. All antimicrobial discs were purchased from Oxoid Chemical Co. UK.

\section{Assessment of combination therapy by checkerboard assay for treatment of multi-drug resistant bacterial isolates}

The minimum inhibitory concentration (MICs) of colistin (COL), imipenem (IMP) and meropenem (MERO) for the bacterial isolates were determined by the broth microdilution method, according to the CLSI [13]. The synergy study for COL/IMP and COL/MERO combinations was carried out for thirty isolates, using the checkerboard method. The checkerboard test was done on 96-well microtiter plates. Most of the strains were resistant or intermediate to carbapenems and susceptible to colistin, the concentration of colistin ranged from 1/32x MIC to 32x MIC while that of the carbapenem (imipenem or merpenem) ranged from $1 / 8 \mathrm{x}$ MIC to $8 \mathrm{x}$ MIC. The initial bacterial inoculum was adjusted to $10^{6} \mathrm{CFU} / \mathrm{ml}$. The plate was incubated for 18 $\mathrm{h}$ at $35^{\circ} \mathrm{C}$. The fractional inhibitory concentration index (FICI) was calculated using the concentrations in the first non-turbid (clear) well in each row and column along the turbidity/non-turbidity interface and then averaged. The results were then classified as: synergy for $\Sigma$ FIC $\leq$ 0.5 ; additive for $\Sigma$ FIC between 0.5 and 1.5 and indifference for values of $\Sigma$ FIC between 1.5 and 2; Antagonism was linked to values above 2 [14-16].

\section{Statistical Methods}

Statistical analysis of checkerboard assay results was made using the chi-square test. Differences were considered significant when $\mathrm{p} \leq$ 0.05 . The data was coded and entered using the statistical package SPSS version 15 (IBM, New York, United States).

\section{Results}

Three hundred and seventy five isolates of $A$. bumannii were identified and confirmed morphologically, by different biochemical tests. All isolates reacted positively to catalase and ability to grow on $44^{\circ} \mathrm{C}$. All isolates were confirmed by API $20 \mathrm{E}$ system showed $90.32 \%$ identification. All isolates were confirmed by PCR assay using $A$. bumannii specific primers. Peaks for positive samples appeared at (750 bp) as shown in Figure 1.

The antimicrobial susceptibility patterns of three hundred and seventy five isolates were examined for antibiotics resistance. Hundred percent of A. bumannii isolates were sensitive to colistin, followed by tobramycin (30.9\%), gentamicin (22.4\%), trimethoprim/ sulfamethoxazol (20\%), tetracycline (18.7\%), piperacilin-tazobactam (13\%), levofloxacin (10.7\%), amikacin $(9.3 \%)$, ciprofloxacin $(9.3 \%)$, meropenem (9.3\%), ampicillin-sulbactam (6.7\%), imipenem $(5.6 \%)$, piperacillin (5\%), ceftazidime (4\%), cefepime (4\%), ceftriaxone $(1.9 \%)$ and $100 \%$ resistant to ceftizoxime.

PCR reactions for confirmed A. bumannii isolates were done for detection of OXA 23,24, 51 and 58 genes that are responsible for carbapenem resistance. Three hundreds and fifteen isolates (84\%), 132 isolates $(35.2 \%), 327$ isolates $(87.2 \%)$ showed positive results to the presence of OXA 23, OXA 24 and OXA 51, respectively and confirmed for the presence of the genes by showing bands on 501, 249, $353 \mathrm{bp}$. No isolates show positive results to the presence of OXA 58 as illustrated in Figure 2.

Minimum inhibitory concentrations determination of colistin, imipenem and meropenem against $A$. bumannii

Thirty strains of A. bumannii of different susceptibility profiles 
Citation: Elhabibi T, Ramzy S (2017) Effect of Antibiotic Combinations on the Sensitivity of Carbapenem Resistant Acinetobacter baumannii Strains. J Microb Biochem Technol 9:132-137. doi: 10.4172/1948-5948.1000356

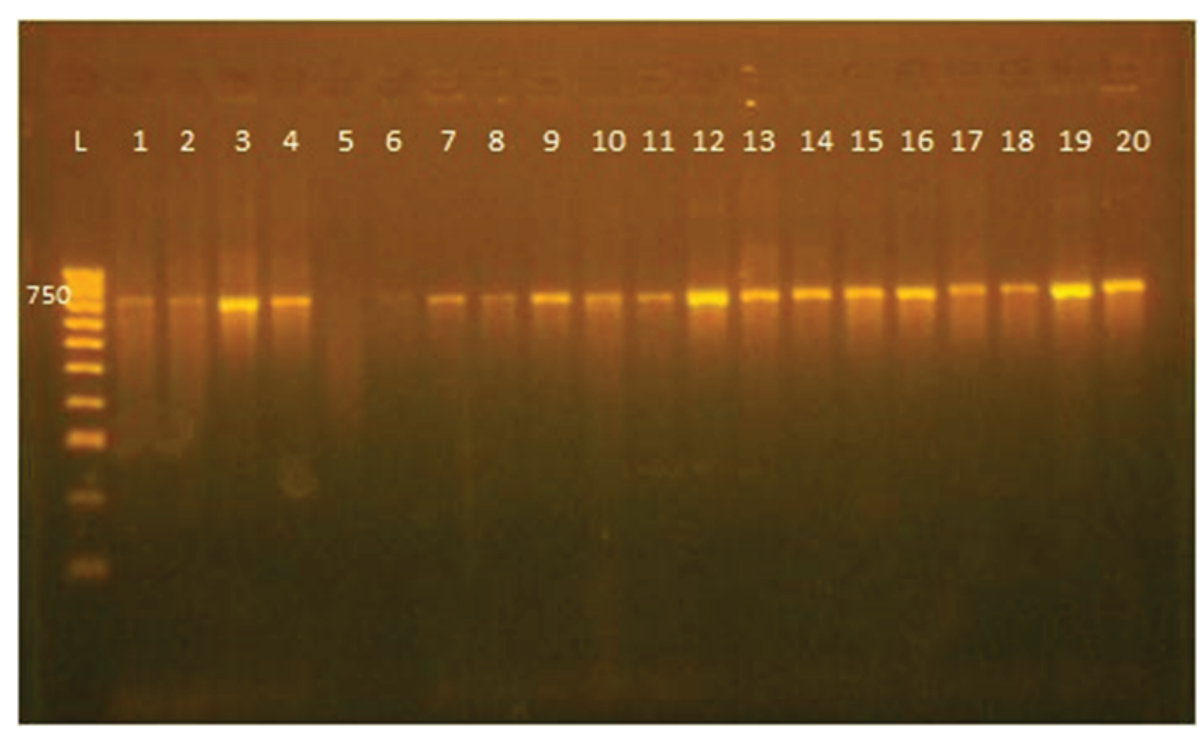

Figure 1: PCR amplification with 16S rRNA gene primers. A 750 bp of $16 S$ rRNA gene. Lane (L), DNA molecular size marker (1500 bp ladder) and Lanes (1-4) and (6-20) show positive result with positive bands of $750 \mathrm{bp}$. Lane (5) shows negative result.

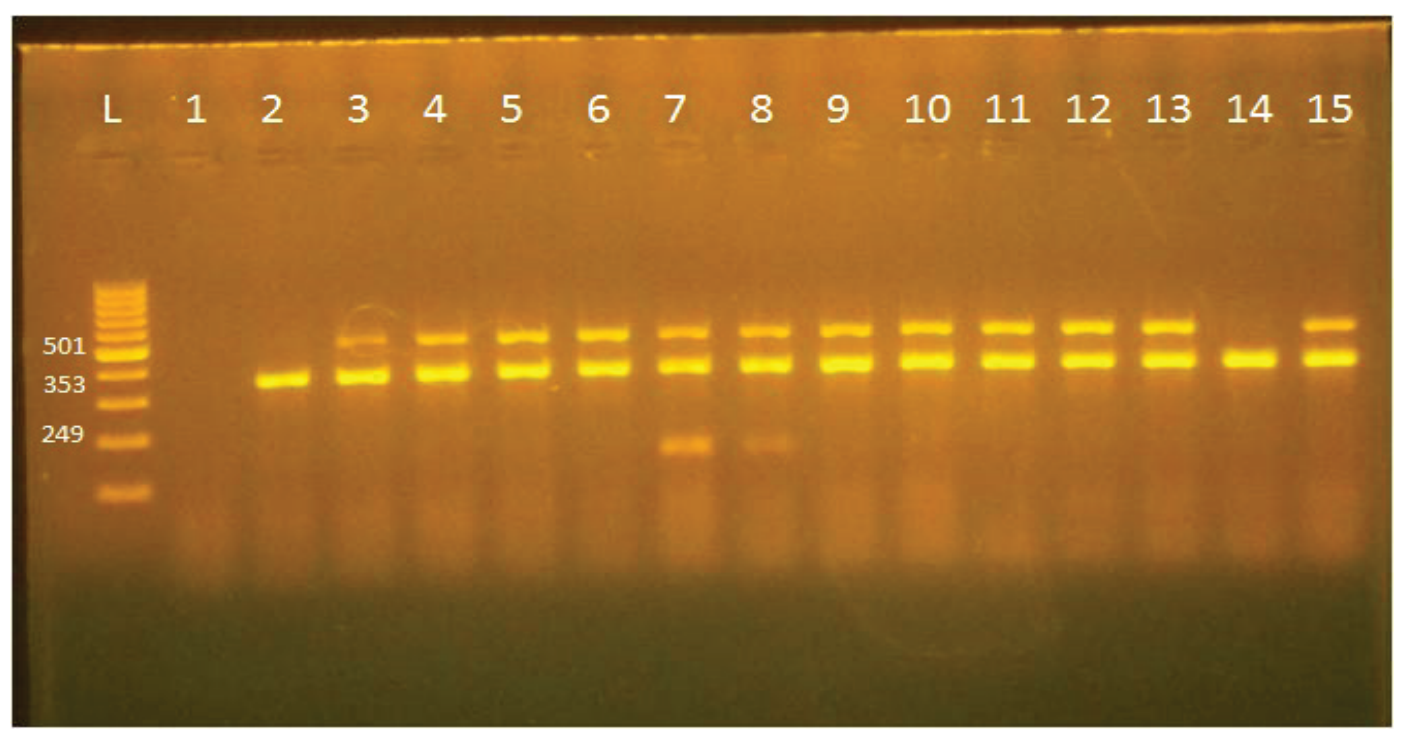

Figure 2: PCR multiplex PCR amplification with OXA genes primers. OXA 23 at 501 bp, OXA 24 at 249 bp, OXA 51 at 353 bp and OXA 58 at 599 bp. Lane (L), DNA molecular size marker (1500 bp ladder) and Lanes (2-15) show positive results for OXA 51 at 353 bp, Lanes (3-13) and (15) show positive results for OXA 23 at 501 , Lane (7 and 8 ) shows positive results for OXA 24 at $249 \mathrm{bp}$.

were used in this test. Most of the strains were resistant or intermediate to carbapenems and susceptible to colistin. The MICs obtained for each antibiotic are shown in Tables 1 and 2. All A. bumannii strains were sensitive to colistin. 12 strains were resistant, 12 strains were intermediate and 6 strains were sensitive to imipenem, 10 strains were resistant, 16 strains were intermediate and 4 strains were sensitive to meropenem.

Results (MIC done in triplet) by broth macrodilution for colistin (COL) for $30 \mathrm{~A}$. bumannii strains shows $100 \%$ sensitive, as 24 isolates showed MIC of 2 and 6 isolates showed MIC of 1. Colistin MIC Interpretive Criteria ( $\leq 2=$ Sensitive and $\geq 8=$ Resistant) [13].

Results (MIC done in triplet) for $30 \mathrm{~A}$. bumannii strains by broth macrodilution for imipenem (IMP) showed 20\% sensitive, $40 \%$ intermediate and $40 \%$ resistant. While for meropenem (MERO) showed $33.3 \%$ sensitive, $53.3 \%$ Intermediate and $13.4 \%$ resistant as shown in Tables 1 and 2.

\section{Checkerboard results}

Tables 2 shows the Fractional inhibitory concentration (FICs) calculated for all A. baumannii strains using the 2 combinations of antibiotics. For the combination of imipenem and colistin 4 of the 30 strains showed synergy with a percentage of $13.3 \%$. While 26 strains showed additive results with a percentage of $86.7 \%$. For the combination of meropenem and colistin on A. baumannii, 20 of the 


\begin{tabular}{|c|c|c|c|c|c|}
\hline \multicolumn{3}{|c|}{ Imipenem } & \multicolumn{3}{|c|}{ Meropenem } \\
\hline No. of Isolates & MIC ug/mI (Median) & Interpretation & No. of Isolates & MIC ug/ml (Median) & Interpretation \\
\hline 5 & 32 & $\mathrm{R}$ & 2 & 16 & $\mathrm{R}$ \\
\hline 3 & 16 & $\mathrm{R}$ & 4 & 32 & $\mathrm{R}$ \\
\hline 4 & 32 & $\mathrm{R}$ & 4 & 32 & $\mathrm{R}$ \\
\hline 12 & 8 & I & 16 & 8 & 1 \\
\hline 2 & 0.125 & $S$ & 1 & 0.125 & $S$ \\
\hline 3 & 0.25 & $S$ & 2 & 0.25 & $S$ \\
\hline 1 & 0.125 & $S$ & 1 & 0.125 & $S$ \\
\hline
\end{tabular}

Imipenem MIC Interpretive Criteria ( $\leq 4=$ Sensitive, $8=$ Intermediate and $\geq 16=$ Resistant), meropenem MIC Interpretive Criteria $(\leq 4=$ Sensitive, $8=$ Intermediate and $\geq$ 16=Resistant) (CLSI, 2016)

Table 1: MICs results by broth macrodilution of imipenem (IMP) and meropenem (MERO) for 30 A. bumannii strains, (MIC done in triplet).

\begin{tabular}{|c|c|c|c|c|}
\hline \multicolumn{2}{|c|}{ Colistin and Imipenem combination } & \multicolumn{2}{c|}{ Colistin and Meropenem combination } \\
\hline No. of isolates & Percent & Effect & No. of isolates & Percent \\
\hline 4 & $13 \%$ & Synergism & 20 & $66.7 \%$ \\
\hline 26 & $87 \%$ & Addition & 10 & $33.3 \%$ \\
\hline
\end{tabular}

Table 2: The FICs calculated for $A$. baumannii strains using different combinations of colistin and imipenem.

30 strains showed synergy with a percentage of $66.7 \%$ while 10 strains showed additive results with a percentage of $33.3 \%$.

The antibacterial effect of both combinations on A. baumannii showed mostly synergistic or additive results. To a significant extent meropenem and colistin showed a better synergy when compared to imipenem and colistin.

\section{Discussion}

Hospital-Acquired Infections (HAIs) are a major cause for high mortality rates worldwide and considered a prominent dilemma in health care facilities. The highest rates of HAIs are highly noticed in ICUs [17].

In the current study a total of 375 non-replicated clinical isolates were collected from various clinical specimens. The antimicrobial susceptibility testing of the isolates was performed using the Kirby Bauer disk diffusion method following the definition of the Clinical and Laboratory Standards Institute using antibiotic discs [13]. Results are consistent with results obtained by AlBshabshe et al. [18] and Altun et al. [19] where all isolates were susceptible to colistin. While in a previous study by Ahanjan et al. [20] showed that susceptibility for colistin was $(65 \%)$ and resistance was seen for cefotaxime (100\%) and ceftazidim (100\%). In a previous hospital study presented by Gupta et al. [21] resistance rate among A. baumannii showed high incidence of resistance was recorded for piperacillin (55\%), followed by ceftriaxone (46\%) and ceftazidime (46\%). This difference may came from the excessive use of colistin in some hospitals that lead to the emergence of colistin resistant strains of $A$. baumannii [21].

Carbapenems are considered the drug of choice for the treatment of infections caused by Multi drug resistant Gram-negative bacilli [22]. In recent years, Egypt has been considered among the countries that reported high rates of antimicrobial resistance [23]. However, carbapenem resistance has been observed repeatedly in non-fermenting bacilli (A. baumannii). Resistance to carbapenem is principally due to carbapenem hydrolyzing enzymes carbapenemases. In the last decade, several classes of $\beta$-lactamases have been detected in A. baumannii [24,25]. The carbapenemases found are mostly belonging to the carbapenem-hydrolyzing OXA-type class D ß-lactamase (CHDL) [26].

Different families of class D B-lactamase (CHDL) have been reported from numerous geographical areas so far. The most common type of enzymes capable of hydrolyzing carbapenems have been reported in A. baumannii, belonging to class D (blaOXA-23-like, blaOXA-24-like, blaOXA-51-like, blaOXA-58-like) [27]. The present study revealed that $(87.2 \%)$ of the carbapenem resistant $A$. baumannii isolates were confirmed for the presence of OXA 51 gene, (84\%) for OXA 23 gene, (35.2\%) for OXA 24 gene. No isolates have revealed the presence of OXA 58 gene. While Al-Hassan et al. [27] revealed that three acquired class D carbapenemases (OXA-23 in $72 \%, O X A-40$ in $4 \%$, OXA-58 in 20\%) were identified among studied carbapenem-resistant A. baumannii strains in two Egyptian centers. Also, our results were nearly confirmed by Gao et al. [28] who confirmed that (100\%) of the carbapenem resistant isolates showed positive results for the presence of OXA 51 gene, (94\%) for OXA 23, and the other two resistance genes (OXA 24 and OXA 58) were not detected in any of the strains. Same results were revealed by Rolain et al. [29] in Hamad hospital in Qatar, that conveyed that $(100 \%)$ of A. baumannii isolates were positive for OXA-23; none were positive for OXA-24, OXA-58 [30].

In Saudi Arabia, OXA-23, OXA-24 and OXA-58 were detected in $72.5,45$ and $37.5 \%$, respectively in isolated A. baumannii strains [31]. OXA-23 was detected in Riyadh and the Eastern Province, with 53 and $79.5 \%$ respectively among non-susceptible $A$. baumannii [32]. In the United Arab Emirates, one study found OXA-23 gene in all isolates under study. In Abu Dhabi, it was found that the OXA-23 gene was detected in $73.6 \%$ of all strains involved in the study by Mugnier et al. [33].

Due to the deficiency or ineffectiveness of infection control programs in many clinics, random/extensive use of antibiotics and many other reasons, resistance greatly appeared within these pathogens and they became acknowledged as highly resistant organisms [32]. Carbapenem resistant A. baumannii strains are currently broadly spread.

Carbapenems were considered as the choice of extreme cases of resistance. With the rise in resistance against carbapenems, most of the available antimicrobial agents are becoming inadequate [34,35]. Physicians have imperfect solution for the treatment of such infections. The old antibiotic colistin, in spite of its toxicity and side effects, is considered today as the last means when these multi-drug organisms are observed [36,37]. The idea of combining colistin with other antibiotics pursuing a synergistic activity, and possibly a less toxicity, looks encouraging. 
Combination therapy limits and decrease microbial resistance, lowers antibiotic toxicity, covers a wide range of pathogens with greater effectiveness and leads to synergy $[38,39]$. One of the aims of this study was to assess the antibacterial activity of the combination of two antibiotics: colistin and a carbapenem (meropenem or imipenem) on A. baumannii using Checkerboard technique.

Most A. baumannii strains are becoming multidrug resistant; the main concern is being resistance to carbapenems [40]. All the strains used in this study were resistant or intermediate to carbapenems however susceptible to colistin. It has been detected that meropenem has greater in vitro efficacy than imipenem against A. baumannii [13].

The present study showed that $(66.7 \%)$ of A. baumannii isolates showed synergistic effect to meropenem/colistin combination and (33.3\%) showed an additive effect. On the other hand, (13\%) of $A$. baumannii isolates showed synergistic effect to imipenem/colistin combination and (87\%) showed an additive effect. These results are consistent with results obtained by Daoud et al. where (54.5\%) of $A$. baumannii isolates showed synergistic effect to meropenem/colistin combination and (45.5\%) showed an additive effect [41]. While, (11\%) of $A$. baumannii isolates showed synergistic effect to imipenem/colistin combination and (89\%) showed an additive effect.

\section{Conclusion}

The best synergy rate and consequent highest antibacterial activity were revealed for the combination of meropenem and colistin. This proposes that the combination could be a good alternative for the treatment of A. baumannii infections until the successful development of a better antibiotic agent. The cause for the increased synergy with meropenem than imipenem might be that most OXA carbapenemases target with better affinity imipenem as compared to meropenem $[42,43]$. Besides, the synergistic or additive effect might be affected by the ability of colistin to disrupt the bacterial outer membrane and raise its permeability for carbapenems and therefore cease the cross linking of the new produced polymers [44,45].

Another benefit for combination therapy is delaying the emergence of bacterial resistance and specifically the promptly developing resistance toward colistin [7]. It must be declared that not only synergy is considered as a benefit for the therapy but also additive result is by itself beneficial, because even a miniature increase in the antibacterial activity using the combination therapy may help clinical success and recovery.

\section{References}

1. Brusaferro S, Arnoldo L, Cattani G, Fabbro E, Cookson B, et al. (2015) Harmonizing and supporting infection control training in Europe. J Hosp Infect 89: $351-356$

2. Obiero WC, Seale AC, Berkley JA (2015) Empiric treatment of neonatal sepsis in developing countries. Pediatr Infect Dis J 34: 659-661.

3. Fouad M, Attia AS, Tawakkol WM, Hashem AM (2013) Emergence of carbapenem-resistant Acinetobacter baumannii harboring the OXA 23 carbapenemase in intensive care units of Egyptian hospitals. Int $\mathrm{J}$ Infect Dis 17: $1252-1254$

4. Talaat M, E-Shokry M, El-Kholy J, Ismail G, Kotb S, et al. (2016) National surveillance of health care-associated infections in Egypt: Developing a sustainable program in a resource-limited country. Am J Infect Control 44: 1296-1301.

5. Farid S, Abouelela A, Eliwa M (2016) Doxycycline and co-trimethoxazole: A new combination for treatment of MDR Acinetobacter baumannii. Does it work? $\mathrm{J}$ Curr Microbiol App Sci 5: 157-164.

6. Nageeb W, Kamel M, Zakaria S, Metwally L (2014) Phenotypic characterization of Acinetobacter baumannii isolates from intensive care units at a tertiary-care hospital in Egypt. EMHJ 20: 203-211.
7. Falagas ME, Karageorgopoulos DE (2008) Pandrug resistance (pdr), extensive drug resistance (xdr), and multidrug resistance (mdr) among gram-negative bacilli: Need for international harmonization in terminology. Clin Infect Dis 46 1121-1122.

8. Schreckenberger PC (2003) Acinetobacter, achromobacter, chryseobacterium, moraxella and other non-fermentative gram-negative rods, pp: 749-779.

9. Sambrook J, Fritsch E, Maniatis T (1989) Molecular cloning: A laboratory manual, second edition. Cold Spring Harbor Laboratory Press.

10. Misbah S, Hassan H, Yusof MY, Hanifah YA, AbuBakar S (2005) Genomic species identification of Acinetobacter of clinical isolates by $16 \mathrm{~S}$ rDNA sequencing. Singapore Med J 46: 461-464.

11. Woodford N, Ellington MJ, Coelho JM, Turton JF, Ward ME, et al. (2006) Multiplex PCR for genes encoding prevalent OXA carbapenemases in Acinetobacter spp. Int J Antimicrob Agents 27: 351-353.

12. Clinical and Laboratory Standards Institute (2011) performance standards for antimicrobial susceptibility testing, twenty-first informational supplement Clinical and Laboratory Standards Institute.

13. Clinical and Laboratory Standards Institute (2013) performance standards for antimicrobial susceptibility testing; twenty-third informational supplement clinical and laboratory standards institute.

14. Eliopoulos GM, Moellering RC (1982) Antibiotic synergism and antimicrobial combinations in clinical infections. Rev Infect Dis 4: 282-293.

15. Joung DK, Joung $H$, Yang DW, Kwon DY, Choi JG, et al. (2012) Synergistic effect of rhein in combination with ampicillin or oxacillin against methicillinresistant Staphylococcus aureus. Exp Ther Med 3: 608-612.

16. White RL, Burgess DS, Manduru M, Bosso JA (1996) comparison of three different in vitro methods of detecting synergy: Time-kill, checkerboard and e test. Antimicrob Agents Chemother 40: 1914-1918.

17. Babamahmoodi F, Ahangarkani F, Davoudi A (2015) Hospital-acquired infections, bacterial causative agents and antibiotic resistance pattern in intensive care units at teaching hospitals in north of Iran. Int J Med Invest 4 152-160.

18. AlBshabshe A, Martin R, Joseph P, Al Hussein A, Haimour W, et al. (2016) Multidrug resistance Acinetobacter species at the intensive care unit, Aseer Central Hospital, Saudi Arabia: A one year analysis. Asia Pac J Trop Med, pp $1-6$

19. Altun HU, Yagci S, Bulut C, Sahin H, Kinikli S, et al. (2014) Antimicrobial susceptibilities of clinical Acinetobacter baumannii isolates with different genotypes. Jundishapur J Microbiol 7: e13347.

20. Ahanjan M, Kholdi S, Rafiei A (2014) Antibiotic-resistance patterns and frequency of TEM and CTX type extended-spectrum ß-lactamases in Acinetobacter clinical isolates. J Mazandaran Univ Med Sci 24: 32-40

21. Gupta N, Gandham N, Jadhav S, Mishra RN (2015) Isolation and identification of Acinetobacter species with special reference to antibiotic resistance. J Nat Sci Biol Med 6: 159-162.

22. Mendiratta DK, Deotale V, Narang P (2005) Metallo-beta-lactamase producing Pseudomonas aeruginosa in a hospital from a rural area. Indian J Med Res 121: 701-703.

23. Mahmoud BA, Zahran AW, Hindawi RG, Labib ZA, Galal R (2013) Prevalence of multidrug-resistant Pseudomonas aeruginosa in patients with nosocomial infections at a university hospital in Egypt, with special reference to typing methods. J Virol Microb.

24. Poirel L, Naas T, Nordmann $P$ (2010) Diversity, epidemiology and genetics of class D beta-lactamases. Antimicrob Agents Chemother 54: 24-38.

25. Poirel L, Bonnin RA, Nordmann $P$ (2011) Genetic basis of antibiotic resistance in pathogenic Acinetobacter species. IUBMB Life 63: 1061-1067.

26. Mathlouthi N, EISalabi AA, Jomaa-Jemili MB, Bakour S, Al-Bayssari C, et al. (2016) Early detection of metallo-ß-lactamase NDM-1- and OXA-23 carbapenemase-producing Acinetobacter baumannii in Libyan hospitals. Inter J Antimicrob Agents 48: 46-50.

27. Al-Hassan L, El Mehallawy H, Amyes SG (2013) Diversity in Acinetobacter baumannii isolates from paediatric cancer patients in Egypt. Clin Microbio Infect 19: 1082-1088.

28. Gao J, Zhao X, Bao $Y$, Mac $R$, Zhou $Y$ et al (2014) Antibiotic resistance and OXA-type carbapenemases-encoding genes in airborne Acinetobacter baumannii isolated from burn wards. Burns J 40: 295-299.

29. Rolain JM, Loucif L, Al-Maslamani M, Elmagboul E, Al-Ansari N, et al. (2016) Emergence of multidrug-resistant Acinetobacter baumannii producing OXA 23 carbapenemase in Qatar. New Microbes New Infect 11: 47-51. 
Citation: Elhabibi T, Ramzy S (2017) Effect of Antibiotic Combinations on the Sensitivity of Carbapenem Resistant Acinetobacter baumannii Strains. J Microb Biochem Technol 9:132-137. doi: 10.4172/1948-5948.1000356

30. Al-Arfaj AA, Ibrahim A.S.S, Al-Salamah AA (2011) Genetic basis of carbapenem resistance in Acinetobacter clinical isolates in Saudi Arabia. Afr J Biotechnol 10: 14186-14196.

31. Abdalhamid B, Hassan $\mathrm{H}$, Itbaileh A, Shorman M (2014) Characterization of carbapenem resistant Acinetobacter baumannii clinical isolates in a tertiary care hospital in Saudi Arabia. New Microbiol 37: 65-73.

32. Opazo A, Sonnevend A, Lopes B, Hamouda A, Ghazawi A, et al. (2012) Plasmid-encoded PER-7 beta-lactamase responsible for ceftazidime resistance in Acinetobacter baumannii isolated in the United Arab Emirates. J Antimicrob Chemother 67: 1619-1622.

33. Mugnier P, Poirel L, Pitout M, Nordmann P (2008) Carbapenem-resistant and OXA-23 producing Acinetobacter baumannii isolates in the United Arab Emirates. Clin Microbiol Infect 14: 879-882.

34. Karageorgopoulos DE, Falagas ME (2008) Pan drug resistance (PDR), Extensive drug resistance (XDR) and multidrug resistance (MDR) among gram-negative bacilli: Need for international harmonization in terminology. Clin Infect Dis 46: 1121-1122.

35. Araj GF, Avedissian AZ, Ayyash NS, Bey HA, El Asmar RG, et al. (2012) A reflection on bacterial resistance to antimicrobial agents at a major tertiary care center in Lebanon over a decade. J Med Liban 60: 125-135.

36. Rossolini GM, Mantengoli E (2005) Treatment and control of severe infections caused by multi-resistant Pseudomonas aeruginosa. Clin Microbiol Infec: 4: $17-32$.

37. Choi WS, Kim SH, Jeon EG, Son MH, Yoon YK, et al. (2010) Nosocomial outbreak of carbapenem-resistant Acinetobacter baumannii in intensive care units and successful outbreak control program. J Korean Med Sci 25: 9991004.

38. Li J, Nation RL, Milne RW, Turnidge JD, Coulthard K (2005) Evaluation of colistin as an agent against multi-resistant gram-negative bacteria. Intern $\mathrm{J}$ Antimicrob Agents 25: 11-25.

39. Lee K, Yong D, Jeong SH, Chong Y (2011) Multidrug resistant Acinetobacter spp.: increasingly prob- lematic nosocomial pathogens. Yonsei Med J 52: 879-891.

40. Teixeira AB, Martins AF, Barin J, Hermes DM, Pormann DC, et al. (2013) First report of carbapenem resistant Acinetobacter nosocomialis isolates harboring isaba1-blaoxa-23 genes in Latin America. J Clin Microb 51: 2739- 2741.

41. Daoud Z, Mansour N, Masri K (2013) Synergistic combination of carbapenems and colistin against $P$. aeruginosa and $A$. baumannii. Open $\mathrm{J}$ Med Microbiol 3 : 253-258.

42. Santella G, Pollini S, Docquier JD, Almuzara M, Gutkind G, et al. (2011) Carbapenem resistance in Pseudomonas aeruginosa isolates: An example of interaction between different mechanisms. Rev Panam Salud Publica 30: 545-548.

43. Oliver A, Levin BR, Juan C, Baquero F, Blaz-quez J (2004) Hyper mutation and the pre-existence of antibiotic-resistant Pseudomonas aeruginosa mutants: Implications for susceptibility testing and treatment of chronic infections. Antimicrob Agents and Chemo 48: 4226-4233.

44. Walsh TR, Toleman MA, Poirel L, Nordmann P (2005) Metallo-beta-lactamases: The quiet before the storm? Clin Microbiol Rev 18: 306-325.

45. Zhang JP, Zhu W, Tian SN, Chu YZ, Chen BY (2010) Molecular characteristics and resistant mechanisms of imipenem-resistant Acinetobacter baumannii isolates in Shenyang, China. J Microb 48: 689-694. 